\title{
ÉTICA DEL DESARROLLO HUMANO SEGÚN EL ENFOQUE DE LAS CAPACIDADES DE MARTHA NUSSBAUM
}

\author{
ETHICS OF HUMAN DEVELOPMENT ACCORDING TO THE \\ PERSPECTIVE OF THE MARTHA NUSSBAUM'S CAPABILITIES
}

Ángel Gómez Navarro*

\begin{abstract}
RESUMEN
El presente escrito analiza el concepto de desarrollo humano desde el enfoque de las capacidades propuesto por la filósofa norteamericana Martha Nussbaum. Se parte de la necesidad de asumir un nuevo enfoque centrado en la persona como fin en sí misma y alternativo al enfoque basado en indicadores cuantitativos. De este modo se analizan las diez capacidades del desarrollo humano teniendo en cuenta su respectivo marco ético y antropológico que las sustentan. Se toma posición respecto de las críticas al enfoque en cuestión y se concluye con una reflexión sobre la educación como clave de las capacidades.
\end{abstract}

PALABRAS CLAVE:

Capacidades, ética, desarrollo, evaluación, enfoque, antropología, educación.

\begin{abstract}
This document analyzes the concept of human development from the perspective of the capabilities proposed by the American philosopher Martha Nussbaum. Be part of the need to take a new perspective based on the person as an end in itself and an alternative to the perspective based on quantitative indicators. Thus, analyzing the 10th human development capabilities considering their respective effective end anthropological framework which support them. Taking a stand on the perspective criticism and concluding with a reflection on education as capabilities key.
\end{abstract}

\section{KEYWORDS:}

Capabilities, ethic, human development, perspective, anthropology, education.

* Docente Principal de la Universidad Femenina del Sagrado Corazón: agomez@unife.edu.pe 


\section{INTRODUCCIÓN}

La riqueza de las naciones está constituida ante todo por cada uno de sus ciudadanos y por las posibilidades que estos tienen para hacer y ser en sus respectivos contextos diferenciados. Por ello, el deber fundamental del estado consiste en promover el desarrollo de una vida justa y digna para todos. Sin embargo, la dificultad surge en el momento en que se concibe el desarrollo en términos casi exclusivamente económicos, tal como se percibe en numerosos ambientes de políticos, de profesionales y de técnicos implicados directamente con el tema, donde el criterio para evaluar dicho desarrollo humano está determinado por el crecimiento per capita del Producto Bruto Interno (PBI) anual, y sus respectivos indicadores estandarizados y validados por las políticas neoliberales de los gobiernos de turno.

Esta concepción del desarrollo, no sólo no promueve una vida saludable y con sentido para todos, sino que tampoco logra superar las persistentes desigualdades que existen entre los ciudadanos, ni la exclusión social ni la pobreza extrema de los desglobalizados que viven y conviven entre nosotros. Es una concepción que aparece distanciada de las preocupaciones éticas que exigen mirar más allá de los mercantilismos arraigados, tanto en la esfera pública como en la privada.

Desde esta situación, real y concreta, surge la necesidad de plantear no sólo un análisis crítico sobre las razones que justifican la evaluación clásica del desarrollo humano, sino también una toma de posición a favor de un nuevo enfoque que considere la centralidad de la persona humana, que la promueva y libere de las políticas locales y globales de no inclusión, y establezca criterios éticos normativos a favor de las libertades intrínsicamente asociadas a las condiciones reales de una justicia social mínima.

\section{El nuevo enfoque del desarrollo humano}

Es sabido que con el enfoque tradicional cuantitativo, un gobierno cualquiera puede llegar a obtener una alta calificación en su desarrollo sin hacer lo suficiente para superar las desigualdades sociales de su gente. Es decir, podría llegar a tener un "alto crecimiento" de su PBI, a pesar de no ofrecerle a todos sus ciudadanos la igualdad de oportunidades que se merecen, tanto en la educación de calidad, como en la asistencia sanitaria, tal como ha sido el caso reciente de Sudáfrica, el país más desarrollado del continente africano, en donde incluso se llegó a realizar una limpieza de pobres en torno al Mundial de Futbol 2010'.

Martha Nussbaum ${ }^{2}$ ha coincidido con Amartya Sen en plantear un nuevo enfoque del desarrollo humano, el cual tuvo su impacto en el informe lanzado por el Programa de las Naciones Unidas para el Desarrollo (PNUD) desde 19903, en donde se sitúa a las capacidades humanas como centro de evaluación

1 http://www.informador.com.mx/internacional/2010/167099/6/en-sudafrica-habría-limpia-de-pobres.htm

2 Filósofa norteamericana de la Universidad de Chicago, obtuvo su doctorado en la Universidad de Harvard y es, junto a Amartya Sen, fundadora de la Asociación Internacional del Desarrollo Humano y las Capacidades. Es defensora de la enseñanza de las humanidades para la democracia y ha hecho aportes muy significativos a la ética y la filosofía política. El 2012, ha obtenido el Premio Príncipe de Asturias por su dedicación a favor de la justicia social, especialmente de los más pobres y vulnerables de la sociedad.

3 Es sabido que desde 1990 se viene elaborando el informe del Desarrollo Humano el cual ha recibido aceptación entre los teóricos y políticos. El documento señala que se trata de "un proceso en el cual 
alternativa al enfoque clásico de corte utilitarista.

El nuevo enfoque rechaza que el crecimiento del PBI sea suficiente para evaluar la calidad de vida de las naciones, pues deja de lado la justa distribución y las legítimas aspiraciones de las gentes. En tal sentido, Nussbaum y Sen consideran que el desarrollo humano tiene que mirar más a la creación de las condiciones de posibilidad para superar la pobreza y la inequidad, antes que quedarse solo en el estudio cuantitativo de los datos económicos. En efecto, los derechos humanos no pueden ser ejercidos sino existen sus respectivas condiciones sociopolíticas y culturales.

En nuestro medio, se puede percibir por ejemplo, con no poca dificultad, a muchas mujeres que casi siempre están en una situación de desventaja respecto, no sólo de la distribución de los recursos sino también, de las condiciones en el ejercicio de su libertad, pues en la práctica sus derechos son nominales, no reales, lo cual las hace más pobres y, por ende, más vulnerables, ya sea ante las oportunidades laborales como ante el uso de sus derechos personales ${ }^{4}$.

Por ello, los estados tienen la obligación moral de superar las limitaciones de la lógica utilitarista, manifiestamente presentes en las prácticas políticas, públicas y privadas, y no reducir sus responsabilidades a la sola medición de los recursos materiales producidos o por producir, o simplemente evitar la peligrosa inflación económica, pues como bien se ha dicho, "el alza del precio del pan puede regular o equilibrar la oferta y la demanda de pan, pero no resuelve el problema del hambre de la gente".

Al respecto, Amartya Sen ha explicado las limitaciones del enfoque utilitarista al señalar los siguientes aspectos:

"Por ejemplo, los principios utilitaristas, se basan en última instancia, sólo en las utilidades, y aunque por el camino puedan tenerse muy en cuenta los incentivos, es la información sobre la utilidad la que se considera a la larga la única base correcta para evaluar la situación o para evaluar actos o normas. En versión clásica del utilitarismo, tal como la formula Jeremy Bentham, la utilidad es el placer, la felicidad o la satisfacción $\mathrm{y}$, por tanto, todo gira en torno a estos logros mentales. Algunas cuestiones potencialmente, que son aspectos de la calidad de vida que no se reflejan de manera suficiente en las estadísticas del placer, no pueden modificar directamente una evaluación normativa en una estructura utilitarista. Sólo pueden desempeñar un papel indirecto a través de su influencia en las cifras de utilidad (es decir, únicamente en la medida en que puedan influir en la satisfacción mental, el placer

se amplían las oportunidades del ser humano. En principio, estas oportunidades pueden ser infinitas y cambiar con el tiempo. Sin embargo, a todos los niveles del desarrollo, las tres más esenciales son disfrutar de una vida prolongada y saludable, adquirir conocimientos y tener acceso a los recursos necesarios para lograr un nivel de vida decente. Si no se poseen estas oportunidades esenciales, muchas alternativas continuarán siendo inaccesibles (PNUD, 1990:33).

4 Según un estudio del Ministerio de la Mujer y Poblaciones Vulnerables, dado a conocer recientemente en torno al Día Internacional de la Erradicación de la Violencia contra la Mujer, 4 de cada 10 mujeres sufren violencia familiar: http://www.mimp.gob.pe/index.php?option=com contentEview=articleEj$\mathrm{d}=605$ Eltemid $=165$ 
o la felicidad). Por otra parte, al modelo agregado del utilitarismo no le interesa, ni es sensible a la distribución real de las utilidades, ya que solo se fija en la utilidad total de todas las personas consideradas en su conjunto. Esto hace que la base de información sea muy reducida, y esta insensibilidad general es una importante limitación de la ética utilitarista" (Citado por Urquijo, 2007: 50-51).

\section{Las diez capacidades según Martha Nussbaum}

En su libro, "Las mujeres y el desarrollo humano" (2002), Nussbaum presenta su nuevo enfoque basado en una lista de diez capacidades, las cuales podrían ser consideradas como metas generales para lograr una sociedad justa $y$, por ende, una vida realmente humana. Veamos el siguiente resumen basado en lo que nos presenta la propia autora (2002: 120-123):

1) Vida: apunta a garantizar una extensión normal de la vida y evitar la muerte prematura.

2) Salud corporal: incluye también la salud reproductiva y una adecuada alimentación, así como disponer de un lugar adecuado para vivir.

3) Integridad física: contar con libertad de movimiento y seguridad frente a todo tipo de agresiones, como los asaltos violentos, la violencia sexual y familiar. También incluye aquí oportunidades para la satisfacción sexual y para la elección en materia de reproducción.

4) Sentidos, imaginación y pensamiento: implica la capacidad de sentir, imaginar, pensar y de razonar al modo "auténticamente humano". Un modo que se cultiva y configura por medio de una educación adecuada (incluyendo alfabetización, entrenamiento científico y matemático básico, sin que eso signifique que quede agotada en ello). Poder usar la imaginación y el pensamiento para la experimentación y la producción de obras y eventos religiosos, literarios, musicales, según la propia elección. Poder usar la mente con garantías de libertad de expresión tanto en lo político como en lo artístico, así como la libertad de prácticas religiosas.

5) Emociones: capacidad para mantener relaciones afectivas, poder amar a aquellos que nos aman y se preocupan por nosotros, y dolernos por su ausencia, experimentar ansia, gratitud y enfado justificado. Poder tener un desarrollo emocional libre de temores o sucesos traumáticos de abuso o descuido.

6) Razón práctica: capacidad para formarse una concepción de bien y reflexionar críticamente sobre los propios planes de vida (lo que implica proteger la libertad de conciencia y de observancia religiosa).

7) Afiliación: la divide en dos partes:

a. Poder vivir con y para los otros; ser capaz de empatía y de comprometerse en distintas maneras de interacción social. Ser capaz de imaginar la situación del otro (proteger esta capacidad implica proteger las instituciones que construyen y promueven estas formas de afiliación, así como proteger la libertad de expresión y de asociación pública). 
b. Que se den las bases sociales del autorrespeto y la no humillación. Ser tratado como un ser dotado de dignidad e igual valor que los demás. Implica establecer normas contrarias a la discriminación por razón de raza, orientación sexual, etnia, casta, religión y origen nacional.

8) Otras especies: Poder vivir una orientación ecológica, cercana y respetuosa con los animales, las plantas y el mundo natural.

9) Juego: Poder reir, jugar y disfrutar de actividades recreativas.

10) Control sobre el propio entorno: también tiene dos dimensiones:

a. Político: capacidad para poder participar de forma efectiva en las elecciones políticas que gobiernan la propia vida; tener derecho a la participación política y a la protección de la libertad de expresión y de asociación.

b. Material: oportunidad real de ejercer la propiedad (de la tierra y de bienes muebles) en condiciones de igualdad de derechos; tener derecho a buscar trabajo en plano de igualdad con los demás. No sufrir persecuciones y detenciones sin garantías. Poder trabajar como un ser humano, ejercer la razón práctica y entrar en relaciones valiosas de reconocimiento mutuo con los demás trabajadores.

Esta lista de capacidades, que está siempre abierta a los aportes interculturales de las sociedades democráticas, debe ser el fundamento de los principios políticos básicos, que los órganos gubernamentales deberían considerar como garantía constitucional para sus ciudadanos (Nussbaum: 2002), aunque nuestra autora advierte que cada gobierno debe decidir cómo ponerlas en funcionamiento.

Asimismo, Nussbaum plantea una clasificación para su respectiva lista, al considerar que existe un conjunto de "capacidades básicas", o innatas a la persona, que requieren de las oportunidades para ser completamente humanas; existen otras capacidades a las que llama "capacidades internas", que son características propias de las personas; y finalmente, están las "capacidades combinadas", que no son sino las capacidades internas combinadas con adecuadas condiciones externas para el ejercicio de la función.

Nussbaum (2002:128-129), lo precisa de la siguiente manera:

A) "Capacidades Básicas, son el equipamiento innato de los individuos y la base necesaria para el desarrollo de las capacidades más avanzadas. La mayor parte de éstas no se pueden aplicar de inmediato. Nussbaum presenta como ejemplo a un recién nacido que tiene las capacidades de habla, de amor y gratitud, de razón práctica, y la capacidad de trabajar.

B) Capacidades Internas, son estados desarrollados por la persona y constituyen, para ella, condiciones suficientes para el ejercicio de la función respectiva. Esta disponibilidad inmediata de la capacidad a veces se produce por la pura maduración corporal, como la capacidad sexual. Otras veces estas capacidades internas se desarrollan sólo con el apoyo del 
medio circundante, como cuando uno aprende a jugar con otros, a amar. Pero, a partir de cierto punto, están ahí y uno puede usarlas.

C) Capacidades combinadas, pueden ser definidas como las capacidades internas combinadas con condiciones externas adecuadas para el ejercicio de la función. Los ciudadanos de regímenes represivos no democráticos tienen la capacidad interna, pero no la combinada para expresar su pensamiento según su conciencia.

Como se puede notar, dicho enfoque tiene una clara orientación política con tendencia a la justicia social y al reconocimiento de los derechos humanos, lo que nos lleva a interrogar sobre cuál es la concepción ética que sustenta dicho enfoque, cuál es su comprensión de persona humana que le sirve de presupuesto y qué relación existe con los planteamientos de otros autores implicados en el tema ${ }^{5}$.

\section{Justificación del enfoque de las capacidades}

Toda praxis humana, como la actividad política, necesita considerar un conjunto de virtudes que, como elemento central y afín a las sociedades, trascienda lo particular y tradicional, y configure a su vez el sentido de las variadas formas de vida común. Tales virtudes, como la justicia y la equidad, definen el contenido de la ética normativa nussbaumiana, con la que no sólo se logra articular 'lo relativo a' una tradición particular con la universalidad, sino también determinar los ámbitos o las esferas por las que transitan, o deben transitar, las sociedades y sus instituciones. Pues la realización de tales virtudes exigen el reconocimiento de la diversidad de los ámbitos socioculturales pero no implican el rechazo de la necesaria universalidad en relación al bien que se persigue. Como decía Aristóteles: "En general, todos los seres humanos buscan no la forma en que vivían sus ancestros, sino el bien". Al respecto, en el libro coeditado con Amartya Sen, "La calidad de vida" (1996), nuestra filósofa afirma:

"Este es un resultado sorprendente, por lo que se refiere a Aristóteles. Porque es obvio que él no solo era defensor de una teoría ética basada en las virtudes, sinotambién defensor de una descripción objetiva única del bien o florecimiento humano. Se supone que esta descripción es objetiva en el sentido de que se le puede justificar mediante referencia a razones que no se derivan sólo de las tradiciones y prácticas locales, sino más bien de los rasgos humanos que subyacen en todas las tradiciones locales y que se pueden encontrar en ellas, ya sea que se les reconozca o no de hecho en esas tradiciones. Aristóteles evidentemente creía que no había ninguna incompatibilidad entre fundamentar una teoría ética en las virtudes y defender la singularidad y objetividad del bien humano (Nussbaum-Sen, 1996:320).

En consecuencia, a nuestra autora no le interesa tanto establecer reglas o procedimientos formales que orienten

5 Hay que tener en cuenta la influencia del pensamiento aristotélico en las obras de Nussbaum, debido a que no sólo buscó superar las limitaciones de la ética utilitarista, sino también su convicción de que las virtudes son el mejor medio para alcanzar la justicia (y no sólo la felicidad), bien primordial de todos los seres humanos. 
nuestro buen obrar, sino forjarnos un carácter, es decir, un ethos, que nos haga capaces de ser moralmente buenos. Y esto es lo que nos permitiría también evaluar las acciones políticas de las instituciones, precisamente en relación al bien deseable que se persigue ${ }^{6}$. Estas virtudes éticas son como el motor que mueven a las personas y a la sociedad para llevar adelante una vida digna de ser vivida: "Los seres humanos cooperan movidos por un amplio abanico de motivos, entre ellos el amor a la justicia, y en especial a la compasión moral hacia aquellos que poseen menos de lo necesario para llevar una vida decente y digna" (Nussbaum, 2007:64)

Por otro lado, ante la interrogante por la concepción de persona que subyace en el enfoque de las capacidades, tenemos que se reconoce al ser humano como sujeto concreto e histórico, cuyo fin es lograr una vida buena basada en las virtudes, y que, por lo mismo, debe estar al centro de las preocupaciones gubernamentales, pues necesita desarrollar sus capacidades en constante interacción con el entorno social y cultural (Nussbaum, 2007)

Es decir, la visión antropológica de nuestra filósofa toma distancia del presupuesto antropológico del contractualismo de Rawls ${ }^{7}$, pues éste supone que todas las personas, en cuanto ciudadanos, están en condiciones de igualdad por tener las siguientes características de: racionalidad, lenguaje y aptitudes mentales y físicas más o menos equivalentes y que, por lo mismo, son capaces de establecer contratos, de unirse en beneficio mutuo y de una cooperación social definida en términos nétamente económicos. Para Nussbaum, esto implica desconocer a las personas que no cuentan con dichas características, como los discapacitados, quienes no podrían contribuir a la sociedad y quedarían definitivamente excluidos.

Al respecto, en su texto sobre "Las fronteras de la justicia" (2007), critica que: "La capacidad para establecer un contrato, y la posesión de las capacidades que hacen posible el beneficio mutuo en la sociedad resultante, no son condiciones necesarias para ser un ciudadano dotado de dignidad y que merece ser tratado con respeto en un plano de igualdad con los demás" (Nussbaum, 2007:35).

En efecto, suponer que todas las personas tengan las mismas condiciones implica no garantizar un trato justo e igualitario, precisamente a los que no son

6 Elsa Gonzáles considera que la propuesta de Nussbaum sigue una teoría normativa del bien con cinco características que cito a continuación: "es normativa, densa, vaga, no-metafísica y universalista. Esta es una teoría normativa en tanto que será capaz de guiar a las distintas instituciones en la busca de la justicia y equidad social, pero también porque desde ella se podrá cuestionar o criticar sus actuaciones actuales. La denominación de "densa" se debe a que esta teoría se pregunta por los fines y por la forma general y el contenido de la forma humana de vida. Y es vaga porque admite que pueden existir múltiples formas específicas en que tales fines son alcanzados por las distintas concepciones personales o locales. Por otra parte, es no-metafísica, o lo que es lo mismo, es esencialista porque parte de las concepciones que los individuos interpretan y evalúan por sí mismos como propias del ser humano. Finalmente, es universalista puesto que reclama que existe un consenso multicultural en torno a las funciones y capacidades que alcanza a definir, es decir, son compartidas por cualquier cultura, religión o propuesta de bien concreto" (Gonzáles, 2007:94).

7 Según Rawls, "los ciudadanos son libres e iguales, debido a que poseen la capacidad para formarse su propia concepción del bien y la capacidad para tener un sentido de justicia. La primera, les permite ser racionales en tanto pueden perseguir lo que cada uno concibe como una vida valiosa. La segunda, les permite ser razonables en tanto son capaces de respetar los términos equitativos de la cooperación social. Estas características son condiciones necesarias y suficientes para ser miembros normales y plenamente cooperantes de la sociedad" (Rawls 1996: 338). 
poseedores de dichas capacidades. Por ello, Nussbaum introduce el concepto de dependencia en su concepción de persona, pues aunque alguien pueda organizar su propia vida con autonomía, necesita de alguien más para realizarse socialmente, más aun si llega a sufrir alguna discapacidad (debido a un accidente o a una enfermedad), lo cual le hace total o parcialmente dependiente de los demás.

Asimismo, Nussbaum considera que es necesario reconocer la diversidad de las personas, no sólo porque aquellas tienen diferentes necesidades y capacidades, sino también porque un buen número de ellas no tiene igualdad de condiciones respecto de las mayorías. De modo tal, que la diversidad de las personas, en cuanto principio antropológico, puede hacer que ellas accedan a un trato justo y respetuoso.

Por consiguiente, dependencia y diversidad configuran la antropología inclusiva de nuestra filósofa, la cual no va en desmedro de la autonomía relativa que también se busca con la lista de capacidades, pues la persona es fin en sí misma y debe encontrar, junto a otras, formas creativas y concretas de vivir y cooperar, tanto para desarrollar sus capacidades como para hacer uso de las que va adquiriendo. Y esto sólo es posible en la medida que se exija a las instituciones actuar con certera responsabilidad, ya que son ellas las que gobiernan las oportunidades vitales de las personas por completo, y no puede plantearse la justicia si se desconoce esa configuración de poder vigente (Nussbaum, 2007). Aquí es donde las políticas gubernamentales se juegan su legitimación y evaluación en relación a que si contribuyen o no a mejorar la calidad de vida de los ciudadanos, estableciendo derechos sociales mínimos, compatibles con diferentes respuestas para las cuestiones de justicia y distribución.

En este sentido, no es suficiente saber cuán satisfecha está o no la persona, sino más bien qué es capaz de ser y hacer ella realmente, en su respectivo contexto diferenciado. Es decir, se trata de promover la apropiación o empoderamiento desde la igualdad de las libertades y derechos, pero sin coaccionar, sino más bien respetando la autonomía individual. Aquí, el Estado está llamado a dar los espacios necesarios para que los ciudadanos realicen sus respectivas elecciones autónomas, incluso a pesar de que algunas minorías puedan rechazar razonablemente las oportunidades, ya que una vida buena debe estar acorde con la dignidad humana. Sin embargo, existe también la posibilidad de que el Estado intervenga las libertades individuales, cuando son amenazados, por ejemplo, los derechos de los más vulnerables, como los niños, para lo cual será muy importante dar las debidas argumentaciones y establer los consensos democráticos.

Ella misma señala, en "Las mujeres y el desarrollo humano" (2002), que: "Incluso si estamos seguros de saber qué es una vida floreciente y que una función particular desempeña un papel importante en ella, no respetamos a los individuos cuando los obligamos a desarrollar ese funcionamiento. Establecemos una base $\mathrm{y}$, como conciudadanos, presentamos cualquier argumento que tengamos a favor de una elección; sin embargo, la decisión final depende de ellos" (Nussbaum, 2002:87-88).

En suma, tanto la concepción ética como la visión antropológica nussbaumiana se constituyen también en un referente fundamental para las políticas gubernamentales, cuyo objetivo 
insoslayalable es la promoción de una vida realmente digna, y en permanente interacción con el entorno sociocultural y socioecológico. La persona es y será siempre fin en sí misma y portadora de valores, caracterizada por la dependencia y la diversidad, cuya centralidad se define por la capacidad para vivir una vida buena, virtuosa, según la razón práctica. De este modo, la persona está por encima de sus propias capacidades, pues ella misma es como la medida de todas las capacidades: "Así podremos reformular nuestro principio de cada persona como fin, articulándolo como un principio de la capacidad de cada persona" (Nussbaum: 2002, 115).

\section{A MODO DE CONCLUSIÓN: Algunas implicancias educativas del enfoque de las capacidades}

El enfoque de las capacidades de Nussbaum permite evaluar los términos del desarrollo humano, la calidad de vida o el bienestar de la persona, desde aspectos relacionados con las libertades que buscan responder a lo que pueden hacer y ser las personas. El uso evaluativo del enfoque implica reconocer el derecho que tienen los ciudadanos a exigir de sus gobiernos la aplicación de las respectivas políticas sociales con igualdad de oportunidades para todos. Por ello, el deber primario del Estado tiene que ser el establecimiento de un marco equitativo en el que cada ciudadano pueda desarrollar sus capacidades, tanto las internas como las combinadas; y uno de los modos más efectivos de hacerlo es a través de una educación de calidad para todos.

En su texto, "El cultivo de la humanidad" (2005), Nussbaum considera que la educación es una actividad clave y valiosa en sí misma, capaz de generar autoestima, entendimiento mutuo y posibilidades de participación.
La educación tiene el gran potencial para desarrollar y expandir mucho más la lista de capacidades, ofreciendo mayores oportunidades de acceso a los ciudadanos presentes y futuros. Por ello, según nuestro modesto parecer, es inaceptable que dicha educación pueda quedar reducida solo a un mero instrumento que desarrolla competencias útiles con el único propósito de satisfacer las necesidades del mercado, según las políticas utilitaristas dominantes, sino que tiene que responder más bien a las exigencias éticas del desarrollo humano, integral y sostenible, centrado precisamente en las capacidades.

Al respecto, Martha Nussbaum ha denunciado, en uno de sus útimos libros, "Sin fines de lucro" (2010), el deterioro de la educación en humanidades en el nivel universitario, lo que afecta gravemente a la convivencia democrática. Ella misma lo expresa en los siguientes términos: "sedientos de dinero, los estados nacionales y sus sistemas de educación están descartando sin advertirlo ciertas aptitudes que son necesarias para mantener viva a la democracia. Si esta tendencia se prolonga, las naciones de todo el mundo en breve producirán generaciones enteras de máquinas utilitarias, en lugar de ciudadanos cabales con la capacidad de pensar por sí mismos, poseer una mirada crítica sobre las tradiciones y comprender la importancia de los logros y los sufrimientos ajenos" (2010, Introdución).

Desde los últimos años, nuestra filósofa ha venido promoviendo una educación orientada a desarrollar tres capacidades fundamentales para el cultivo de la humanidad y la democracia: pensamiento crítico, ciudadanía global y comprensión imaginativa. Se trata de una concepción que promueve en los estudiantes la adquisición de destrezas 
y habilidades, tanto socio-cognitivas como actitudinales, para contrarrestrar las desigualdades y afirmar las virtudes democráticas (Nussbaum, 2005).

Este modelo educativo, que algunos no han dudado en llamar crítico-socrático (Benéitez, 2008), busca promover una formación para la ciudadanía, no sólo local sino también global, donde las responsabilidades civicas son asumidas según los marcos axiológicos definidos por la igualdad de oportunidades, la libertad participativa, la no discriminación, la autonomía responsable, la tolerancia y el pluralismo.

En suma, el enfoque de las capacidades de Martha Nussbaum, es también un desafío para las políticas educativas que necesitan forjar un ethos personal y social, que haga posible el ejercicio de la ciudadanía responsable y autónoma, capaz de transformar las bases de la sociedad, según una razón práctica que busca la justicia y la dignidad de la persona; y en donde "la idea central es la del ser humano como un ser libre dignificado que plasma su propia vida en cooperación y reciprocidad con otros, y no siendo modelado en forma pasiva o manejado por todo el mundo a la manera de un animal de rebaño" (Nussbaum, 2002:113).

\section{REFERENCIAS}

BENÉITEZ. J. (2009) Martha Nussbaum y la educación socrática para la ciudadanía. Recuperado de: http:// www.revistaeducacion.mec.es/re350/ re350_17.pdf

BONI, A. (2013) Universidad y educación para el desarrollo. Recuperado de: http://www.sextocongresocud.es/
wp-content/uploads/2013/03/A.Boni_ Universidad-y-Epd.pdf

GONZÁLES, E. (2007) "Una lectura actualizada de la ética aristotélica. La mirada de Martha Nussbaum". En Revista Quaderns de filosofía y ciencia, 37, pp.91-100. Recuperado de: http://www.uv.es/sfpv/quadern_ textos/v37p91-100.pdf

NUSSBAUM, M. y SEN, A. (Ed.) (1996) La calidad de vida. México: FCE.

NUSSBAUM, M. (2002) Las mujeres y el desarrollo humano. El enfoque de las capacidades. Barcelona: Herder.

NUSSBAUM, M. (2005) El cultivo de la humanidad: una defensa clásica de la reforma en la educación liberal. Barcelona: Paidós.

NUSSBAUM, M. (2007) Las fronteras de la justicia. Consideraciones sobre la exclusión. Barcelona: Paidós.

NUSSBAUM, M. (2010) Sin fines de lucro. Por qué la democracia necesita de las humanidades. Buenos AiresMadrid: Katz Editores.

RAWLS, J. (1996) El liberalismo Político. Barcelona: Crítica.

SEN, A. (2000) Desarrollo y Libertad. Madrid: Editorial Planeta.

URQUIJO, M. (2007) El enfoque de las capacidades de Amartya Sen: Alcances y límites. Tesis Doctoral sustentada en la Universidad de Valencia. Recuperado de: http://www.tdx. cat/bitstream/handle/10803/9862/ urquijo.29E3F2BE5B489CC55A64F04 $67 \mathrm{CC} 18 . \mathrm{tdx} 2$ ?sequence $=1$ 